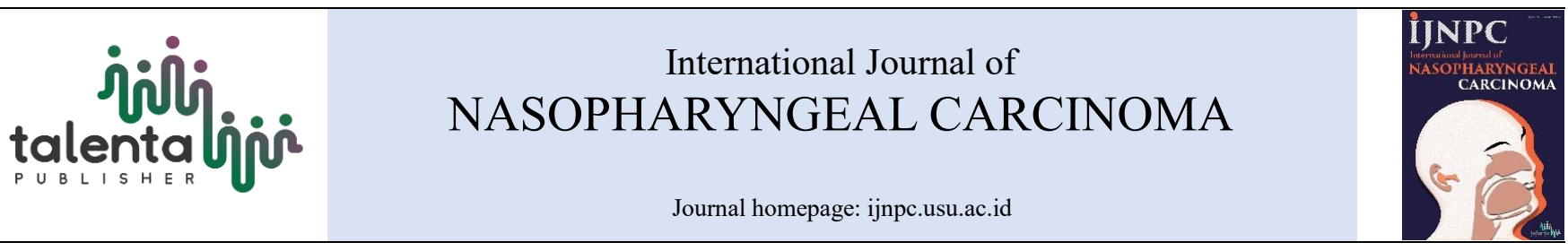

\title{
POST-TREATMENT FOLLOW-UP OF NASOPHARYNGEAL CARCINOMA PATIENTS: A REVIEW
}

\author{
Ika Dewi Mayangsari ${ }^{*}$ \\ ${ }^{I}$ ENT Department, Faculty of Medicine, Universitas Indonesia-Cipto Mangunkusumo Hospital
}

\begin{abstract}
Introduction: Locoregional failure of nasopharyngeal carcinoma (NPC) occurs despite the aggressive treatment that has been done. Optimal and accurate follow-up schedule after the radiotherapy or chemoradiotherapy sessions could improve the patient's quality of life (QoL).

Discussion: Kwong et al. reported complete histological remission within 3 months after therapy and positive histological signs at $>12$ weeks considered a persistent disease. Kong et al. (2017) showed that the pathologica reatment response using CT scan and MRI and stated that the median time to full regressions is 4.9 months. Some studies also reported that primary tumor continuously regressed $>4$ months after treatment and delayed remission $>12$ weeks is not a prognostic factor for poor survival.

The use of MRI is superior to CT scan to detect residual or recurrent NPC. MRI showed good results in differentiating mature scar and tumor, whereas CT scan could not differentiate fibrosis and tumor because of the radiation therapy-induced inflammation. On the other hand, PET-CT has higher sensitivity compared to CT scan and MRI, but poor cost-effectiveness. Various studies and NCCN 2018 guidelines recommend the use of imaging between the third and sixth months after therapy to evaluate treatment response.

Conclusion: Assessment of treatment-related late toxicities also an important factor to improve patient's QoL. Evaluation of nutrition, speech difficulties, swallowing, hearing, dental care should be done annually and peer support groups can help improve patient's QoL.

Assessment of residual disease should be done at the minimum of 10 weeks after the completion of the therapy and long-term follow-up is a must because locoregional recurrences of NPC are common within the first two to three years after the treatment.
\end{abstract}

\section{Article Info}

Keywords:

Nasopharyngeal carcinoma, treatment, post-treatment

\section{*Corresponding author:}

Address: ENT Department Cipto Mangunkusumo Hospital Staff Building $7^{\text {th }}$ fl, Jalan Diponegoro 71, 10430, Jakarta-Indonesia

e-mail: idmayang@yahoo.com

\section{INTRODUCTION}

Nasopharyngeal carcinoma (NPC) is a malignant disease and one of the leading cause of death in several countries, including Indonesia with 7.391 deaths in 2012 [1]. NPC is a highly radiosensitive malignancy and based on the guidelines of the National Comprehensive Cancer Network (NCCN) in 2018, radiation therapy is the treatment of choice for primary NPC and concurrent chemoradiotherapy for locoregionally advanced NPC $[2,3]$. Despite the aggressive treatment, local or regional failure, and/or distant metastasis can occur in some patients. Thus, the accurate timing of evaluation of the treatment response is important in improving the patient's prognosis. Too early in evaluating the treatment response could result in overtreating, whereas too late in evaluating could result in poor salvage treatment outcome [4].

High-dose radiotherapy and concurrent chemotherapy also induce acute and late toxicities. Where late toxicities can occur months, even years later after the treatment is complete. Therefore, close follow-up after the treatment completion is crucial for NPC patients and early detection of locoregional treatment failure is important as limited locoregional persisten or recurrent foci can usually be effectively salvaged [4]. However, there is no definite consensus for the optimal follow-up schedule for non-metastatic NPC patients. This review aims to discuss the optimal follow-up timing after chemoradiation therapy in nasopharyngeal carcinoma patients.

\section{MAIN TEXT}

\subsection{Pattern of Pathological Response to Treatment}

Several studies reported that the cut-off times for treatment response vary from 1 to 4 months after a full course of radiotherapy or concurrent chemoradiotherapy. A study from Kwong DL et al. used serial biopsies to evaluate tumor regression patterns. As 803 NPC patients who completed radiotherapy treatment, underwent serial biopsies every 2 weeks for 3 months, they stated that 617 patients $(76.8 \%)$ showed negative histology in the first session and 186 patients $(23.2 \%)$ showed continuous spontaneous remission on repeat biopsies. In the end, 55 patients had persistent disease, 3 months after the treatment. In the total of $93.2 \%$ of the patients showed complete histological remission within 3 months after the radiation therapy. In this study, they use 12 weeks cut off, if the positive biopsy remains after 12 weeks, it considered persistent disease [5].

On the other hand, a study by Kong M et al. (2017) [6], showed that the median time to full regression after completion of therapy was 4.9 months. They used a CT scan and/or MRI to evaluate the tumor regression. 18 patients showed full regression after 3 months of the therapy and only 8 patients showed full primary tumor regression within 3 months of the therapy. Some possible reasons for the different patterns of tumor regression between these two studies are the frequency and the way of evaluating the treatment response. Kwong DL et al. Study [5] started the evaluation from 2 weeks after the treatment completion, whereas Kong $\mathrm{M}$ et al. evaluate every 1 to 2 months after the treatment completion. This could be the reason why the primary tumor regression detected later than the actual occurrence in M Kong et al. Study [6]. Besides, the histological process begins immediately after the exposure of the radiotherapy whereas clinical features may not significantly change immediately after the therapy, yet it will become apparent weeks or months after the exposure of the radiotherapy [6].

Study by Lin et al. (2013) [7] stated that primary tumor eradication could continuously regressed for $>4$ months after the completion of chemoradiotherapy and delayed tumor regression is not associated with poor prognosis (Lv JW, 2017) [8]. Biopsies less than 3 months from the completion of the treatment showed a high rate of false positive. Several studies recommend waiting for at least 3 to 6 months and several studies recommend waiting for $>4$ months after the treatment completion for full regression of NPC if there are no signs of persistent or recurrent disease on follow-up sessions. 


\subsection{Detection of Local, Regional, and Distant Treatment Failures and Recurrences}

\subsubsection{Clinical Examination}

A thorough history taking and physical examinations have to be done during each follow-up sessions. History taking regarding signs and symptoms of recurrence NPC should be asked. Some patients with recurrence did not have specific symptoms and were found only at routine examinations. While most patients have symptoms such as bloody nasal discharge, headache, tinnitus, neck mass, nasal obstruction, hearing loss, blurred vision, facial edema, a sense that ears were plugged, epiphora, facial numbness, and abducens dysfunction. A study by Li JX et al. (2012) stated that nasal bloody discharge and headache are the most common symptoms for recurrent nasopharyngeal carcinoma [9].

\subsubsection{Endoscopy}

The use of nasopharyngoscopy after completion of radiotherapy treatment for evaluating local failure remains unclear. A study by Ragab et al. (2008) [10] showed that rigid endoscopy was useful for NPC's post-treatment follow-up with sensitivity, specificity, positive predictive and negative predictive values of $66.6 \%, 95 \%$, $66.6 \%$, and $95 \%$ respectively. High specificity and negative predictive value imply that endoscopic examination is good for the detection of residual or recurrent NPC Whereas Kwong DL et al. (2001) study showed that the use of flexible endoscopy to detect recurrent NPC had low sensitivity, specificity, positive predictive and negative predictive values of $40.4 \%, 84.4 \%, 16.3 \%$, and $95 \%$ [4].

A study by Bagri et al. (2013) [11] also showed high sensitivity, specificity, positive predictive and negative predictive values for rigid endoscopy examinations for post-treatment NPC patients; they were $77.78 \%, 93 \%, 70 \%$, and $95.24 \%$ respectively. Several studies stated that the rigid endoscopy is better than flexible endoscopy to detect residual or recurrent NPC after the treatment completion. It is because rigid endoscopy gives a wider field, better illumination, excellent resolution, and sharper image compared to flexible endoscopy.

A study by Wang WH, 2011 reported the detection of mucosal recurrent nasopharyngeal carcinoma using white-light (WL) endoscopy compared to narrowband imaging (NBI). All suspected lesions detected by WL endoscopy were seen as granular or ulcerative lesions, whereas suspected lesions detected by NBI were seen as a brownish area with scattered brown spots. The study stated that the use of NBI endoscopy could improve the detection of recurrent mucosal NPC compared to WL endoscopy with sensitivity, specificity, positive predictive value, and negative predictive value fo WL endoscopy were $37.5 \%, 92.9 \%, 30 \%, 94.8 \%$ respectively. On the other hand, the results of recurrent NPC diagnosis by NBI view were as follows: sensitivity $87.5 \%$, specificity $74.5 \%$, positive predictive value $21.9 \%$, and negative predictive value $98.6 \%[12]$.

In contrast, a study by $\mathrm{Ng}$ et al. (1999) stated that $28 \%$ of deep-seated recurrent NPC were not detected on endoscopy and it was diagnosed by magnetic resonance imaging (MRI). Recurrences in the submucosa or deeper structures are not accessible by endoscopy and the sensitivity decreases. Therefore, the use of endoscopy alone for early detection of local recurrence may not be sufficient [4].

\subsubsection{Imaging for Locoregional Recurrence}

Imaging modalities that are commonly used for post-treatment follow-up are computed tomography (CT) scan, magnetic resonance imaging (MRI), and positron emission tomography (PET) scan. The use of head and neck CT scan after the treatment completion is common. However, the clinical value and optimal timing for $\mathrm{CT}$ scan are still debatable. CT scan cannot differentiate between post-treatment fibrosis, inflammation, edema from post-radiotherapy changes and residual or recurrent tumor $[7,8]$.

Gong et al. study reported that MRI was superior to CT to detect residual or recurrent NPC after treatment. MRI is great for differentiating mature scar tissue and tumor. However, the highest rates of false positive occur within one month after the radiation therapy. It was hypothesized that the inflammation response by radiation therapy caused a false positive diagnosis. Several studies stated that the inflammation would gradually disappear after the first three months from the completed therapy. Therefore, lots of studies and NCCN 2018 also recommend the use of imaging between the third to sixth months after the completion of the therapy to reduce false positive diagnosis [2].

PET-CT has higher specificity than MRI and less influenced by inflammation caused by radiation therapy and higher sensitivity ( $95 \%)$ compared to CT (76\%) and MRI (78\%) in detecting locally residual or recurrent tumor [8] Study by Yen et al (2006) [13] stated that PET has high sensitivity for detecting local, regional, and distant recurrences $(91.6 \%, 90 \%$, and $100 \%$ repectively) and high specificity ( $76 \%, 89 \%$, and $90.6 \%$ respectively). Despite the high sensitivity and specificity, CT/MRI are preferred than PET scan because of the high cost. Yen et al. (2009) [14] suggested the use of PET or PET-CT as an additional imaging modality if CT or MRI showed uncertainly result [1]. The importance to assess the locoregional recurrence related to the evaluation of treatment success rate. The proper time in identification of locoregional recurrence could enhance the quality of life $(\mathrm{QoL})$ due to the prognosis improvement. [4].

\subsubsection{Imaging for Distant Metastases}

A study by Li AC, et al. (2015) [15], the most common site for nasopharyngeal carcinoma distant metastases are bone, lung, and liver respectively. The diagnosis can be made using multiple imaging modalities, such as chest c-ray and/or CT scan for the thorax, ultrasound and CT of the abdomen for liver metastases, and $\mathrm{x}$-ray and/or bone scan for bone metastases. A study by Yen et al. (2006) [13] stated that Pet scan or PET/CT has higher sensitivity for diagnosing metastases in NPC compared to conventional work-up modalities. However, because of the poor cost-effectiveness of PET/CT, conventional workup modalities are still widely used nowadays $[16,17]$.

NCCN guidelines recommend the use of imaging modalities (PET/CT or conventional modalities) for distant metastasis work-up in high-risk NPC patients, including nonkeratinizing histology, stage III-IV disease, N2-N3 disease, and endemic phenotype [2]. Caglar et al. (2003) reported the use of bone scintigraphy prior to therapy and at 1 -year intervals to evaluate bone metastasis [18].

\subsubsection{Plasma EBV DNA \& EBV Serology Measurement}

Plasma EBV DNA is one of the examinations included in the follow-up routine. A study by Lv JW, et al. (2017) [8] reported that detectable plasma EBV DNA at three months after the treatment completion was associated with the presence of tumor residue. It was found that the patients are prone to have residual tumor, local and distant failure if there is a detectable EBV DNA after the treatment. It was hypothesized that the plasma EBV DNA level represents the tumor DNA level [19].

\subsubsection{Assessment of Treatment-Related Late Toxicities}

Assessment of the late toxicities related to radiation therapy also has to be done in the routine follow-up since late toxicities can occur weeks, months even weeks after the completion of the treatment. NPC patients often have difficulties in speech or hearing and swallowing, hence swallowing evaluation and rehabilitation are clinically indicated for post-treatment NPC patients. Sensorineural hearing loss also can be expected after platinum-based chemotherapy. Thyroid function screening tests also need to be conducted because radiation therapy could cause hypothyroidism. NCCN 2018 recommend checking thyroid-stimulating hormone (TSH) every 6 to 12 months after neck radiation therapy. Dental also care one of the important examinations because of osteoradionecrosis as one of the dental adverse events. And last but not least is patient support groups for cancer survivors because peer support also can improve quality of life of the patients and families [4].

\section{CONCLUSION}

Early detection of residual or recurrence NPC allows more treatment options to be considered. However, assessment of residual disease should be done at the minimum of 10 weeks after the completion of the therapy and long-term followup is a must because locoregional recurrences of NPC are common within the first two to three years after the treatment.

Table 1. Post Treatment Follow-up Examinations Recommendation

\begin{tabular}{|c|c|c|}
\hline Modality & Tests & Schedule \\
\hline Clinical & $\begin{array}{l}\text { Physical examinations (including } \\
\text { complete head and neck exam), } \\
\text { Nasoendoscopy (WL and/or NBI } \\
\text { view), Evaluation of speech, hearing, } \\
\text { swallowing, and nutrition as indicated }\end{array}$ & $\begin{array}{l}\text { Year } 1: \text { every } 1-3 \text { months } \\
\text { Years } 2-3: \text { every } 3 \text { months } \\
\text { Years } 4-5: \text { every } 6 \text { months } \\
>5 \text { years: every } 12 \text { months }\end{array}$ \\
\hline Laboratory & $\begin{array}{l}\text { TSH, free FT 4, Other endocrine tests } \\
\text { as indicated, Plasma EBV DNA } \\
\text { (optional) }\end{array}$ & $\begin{array}{l}\text { Every } 6-12 \text { months } \\
3 \text { months after therapy }\end{array}$ \\
\hline Imaging & $\begin{array}{l}\text { MRI of the head and neck, Chest x-ray, } \\
\text { CT of the thorax/abdomen, liver USG, } \\
\text { bone scan, PET/CT scan when } \\
\text { clinically indicated (metastases or } \\
\text { uncertain result) }\end{array}$ & $\begin{array}{l}3 \text { months after therapy or within } \\
6 \text { months after therapy, then } \\
\text { annually (when clinically } \\
\text { indicated) High-risk NPC, } \\
\text { annually }\end{array}$ \\
\hline Others & $\begin{array}{l}\text { Smoking cessation and alcohol } \\
\text { counseling, Dental care, Peer support } \\
\text { group }\end{array}$ & $\begin{array}{l}\text { As clinically indicated } \\
3 \text { months following RT }\end{array}$ \\
\hline
\end{tabular}




\section{REFERENCE}

[1] Mahdavifar N, Mohammadian M, Salehiniya H. Gallbladder Cancer in The World: Epidemiology, Incidence, Mortality and Risk Factors. World Cancer Research Journal. 2018;5(3):7.

[2] Colevas AD, Yom SS, Pfister DG, Spencer S, Adelstein D, Adkins D, et al. NCCN Guidelines Insights: Head and Neck Cancers, Version 1.2018. Journal of the National Comprehensive Cancer Network. 2018;16(5):479-90.

[3] Network NCC. NCCN Clinical Practice Guidelines in Oncology: Head and Neck Cancers. 2018.

[4] Lu JJ, Cooper JS, Lee AW. Nasopharyngeal Cancer: Multidisciplinary Management: Springer Science \& Business Media; 2010.

[5] Kwong DL, Nicholls J, Wei WI, Chua DT, Sham JS, Yuen P, et al. The Time Course of Histologic Remission after Treatment of Patients with Nasopharyngeal Carcinoma. Cancer: Interdisciplinary International Journal of the American Cancer Society. 1999;85(7):1446-53.

[6] Kong M, Hong SE. Tumor Regression Patterns by Follow-Up Duration in Patients with Nasopharyngeal Carcinoma Treated with Concurrent Chemoradiotherapy. Journal of Radiation Research. 2017;58(2):232-7.

[7] Lin G-w, Wang L-x, Ji M, Qian H-z. The Use of MR Imaging to Detect Residual Versus Recurrent Nasopharyngeal Carcinoma Following Treatment with Radiation Therapy. European Journal of Radiology. 2013;82(12):2240-6.

[8] Lv J-W, Zhou G-Q, Li J-X, Tang L-L, Mao Y-P, Lin A-H, et al. Magnetic Resonance Imaging-Detected Tumor Residue after Intensity-Modulated Radiation Therapy and Its Association with PostRadiation Plasma Epstein-Barr Virus Deoxyribonucleic Acid in Nasopharyngeal Carcinoma. Journal of Cancer. 2017;8(5):861.

[9] Li J-X, Lu T-X, Huang Y, Han F. Clinical Characteristics of Recurrent Nasopharyngeal Carcinoma in High-Incidence Area. The Scientific World Journal. 2012;2012.

[10] Ragab S, Erfan F, Khalifa M, Korayem E, Tawfik H. Detection of Local Failures after Management of Nasopharyngeal Carcinoma: A Prospective, Controlled Trial. The Journal of Laryngology \& Otology. 2008;122(11):1230-4.

[11] Bagri PK, Singhal MK, Singh D, Kapoor A, Jakhar SL, Sharma N, et al. Diagnosis of Post-Radiotherapy Local Failures in Nasopharyngeal Carcinoma: A Prospective Institutional Study. Iranian Journal of Cancer Prevention. 2014;7(1):35.

[12] Wang W-H, Lin Y-C, Chen W-C, Chen M-F, Chen C-C, Lee K-F. Detection of Mucosal Recurrent Nasopharyngeal Carcinomas after Radiotherapy with Narrow-Band Imaging Endoscopy. International Journal of Radiation Oncology* Biology* Physics. 2012;83(4):1213-9.

[13] Yen T-C, Lin C-Y, Wang H-M, Huang S-F, Liao C-T, Kang C-J, et al. 18F-FDG-PET for Evaluation of The Response To Concurrent Chemoradiation Therapy with Intensity-Modulated Radiation Technique for Stage T4 Nasopharyngeal Carcinoma. International Journal of Radiation Oncology* Biology* Physics. 2006;65(5):1307-14.

[14] Yen R-F, Yen M-F, Hong R-L, Tzen K-Y, Chien C-R, Chen TH-H. The Cost-Utility Analysis of 18-Fluoro-2-Deoxyglucose Positron Emission Tomography in The Diagnosis of Recurrent Nasopharyngeal Carcinoma. Academic Radiology. 2009;16(1):54-60

[15] Li A-C, Xiao W-W, Shen G-Z, Wang L, Xu A-A, Cao Y-Q, et al. Distant Metastasis Risk and Patterns of Nasopharyngeal Carcinoma in The Era of IMRT: Long-Term Results and Benefits of Chemotherapy. Oncotarget. 2015;6(27):24511.

[16] Kapoor A, Kalwar A, Kumar N, Maharia S, Nirban RK, Kumar HS Detection of Bone Metastasis in Nasopharyngeal Carcinoma by Bone Scintigraphy: A Retrospective Study in Perspective of Limited Resource Settings. Clinical Cancer Investigation Journal. 2015;4(1):17.

[17] Saito N, Nadgir RN, Nakahira M, Takahashi M, Uchino A, Kimura F, et al. Posttreatment CT and MR Imaging in Head and Neck Cancer: What The Radiologist Needs To Know. Radiographics. 2012;32(5):1261-82.

[18] Caglar M, Ceylan E, Ozyar E. Frequency of Skeletal Metastases in Nasopharyngeal Carcinoma after Initiation of Therapy: Should Bone Scans Be Used for Follow-Up? Nuclear Medicine Communications. 2003;24(12):1231-6.

[19] Li W-F, Zhang Y, Huang X-B, Du X-J, Tang L-L, Chen L, et al Prognostic Value of Plasma Epstein-Barr Virus DNA Level During Posttreatment Follow-Up in The Patients with Nasopharyngeal Carcinoma Having Undergone Intensity-Modulated Radiotherapy. Chinese Journal of Cancer. 2017;36(1):87. 